JOURNAL OF INTEGRAL EQUATIONS

AND APPLICATIONS

Volume 4, Number 4, Fall 1992

\title{
SPECTRAL APPROXIMATIONS FOR WIENER-HOPF OPERATORS II
}

\author{
P.M. ANSELONE AND I.H. SLOAN
}

ABSTRACT. The comparison of spectral properties of operators

$$
\begin{aligned}
K f(s) & =\int_{0}^{\infty} \kappa(s-t) f(t) d t, \\
K_{\beta} f(s) & =\int_{0}^{\beta} \kappa(s-t) f(t) d t,
\end{aligned}
$$

with $\kappa \in L^{1}(R)$, which was initiated in [3], is extended here in several directions. In [3], the operators were defined on the space of bounded continuous functions on the half-line. Now they are studied on $L^{2}\left(R^{+}\right)$. The spectra are unchanged. Particular attention is paid to the self-adjoint case. There is a very close relationship between spectral properties of $K$ and $K_{\beta}$ as $\beta \rightarrow \infty$. Under further restrictions, $\sigma\left(K_{\beta}\right)$ is asymptotically dense in $\sigma(K)$ as $\beta \rightarrow \infty$. The proofs are based directly on properties of the operators. This enables us to avoid extraneous hypotheses which Fourier transform methods often require.

1. Introduction. In $[\mathbf{3}]$ we investigated the relationship between the spectrum of a Wiener-Hopf operator

$$
K f(s)=\int_{0}^{\infty} \kappa(s-t) f(t) d t, \quad s \in R^{+}=[0, \infty],
$$

and the spectra of the corresponding finite-section operators

$$
K_{\beta} f(s)=\int_{0}^{\beta} \kappa(s-t) f(t) d t, \quad s \in R^{+}, \beta \in R^{+},
$$

where $\kappa \in L^{1}(R)$ and $f \in X^{+}$, the space of bounded, continuous, real or complex functions on $R^{+}$with $\|f\|=\sup |f(t)|$. To avoid trivialities, assume that $\|\kappa\|_{1} \neq 0$. Then $K \neq 0$ and the operator $K$ is not compact. However, the operators $K_{\beta}$ are compact.

We proved in [3] that every neighborhood of $\sigma(K)$ contains $\sigma\left(K_{\beta}\right)$ for $\beta$ sufficiently large and that every point in $\sigma(K)$ is an asymptotic

Copyright (C)1992 Rocky Mountain Mathematics Consortium 
eigenvalue of $K_{\beta}$ as $\beta \rightarrow \infty$. The main purpose of this paper is to clarify further the relationship between spectral properties of $K$ and $K_{\beta}$. The spectra of $K$ and $K_{\beta}$, acting on the space $X^{+}$, are denoted by $\sigma(K)$ and $\sigma\left(K_{\beta}\right)$.

We say that $\sigma\left(K_{\beta}\right)$ is asymptotically dense in $\sigma(K)$ as $\beta \rightarrow \infty$ if, for any $\varepsilon>0$, the $\varepsilon$-neighborhood of $\sigma\left(K_{\beta}\right)$ contains $\sigma(K)$ for $\beta$ sufficiently large. This is true for the Picard kernel $\kappa(u)=e^{-|u|}$. See $[\mathbf{3}]$ for the details. However, it is not true in general. Two counterexamples are given in [3], with $\sigma(K)$ a disc and $\sigma\left(K_{\beta}\right)=\{0\}$ in each case.

If $\sigma\left(K_{\beta}\right)$ is asymptotically dense in $\sigma(K)$ as $\beta \rightarrow \infty$, then $\sigma\left(K_{\beta}\right) \rightarrow$ $\sigma(K)$ as $\beta \rightarrow \infty$ in the sense of the Hausdorff semi-metric for the distance between two sets.

The Fourier transform of $\kappa$,

$$
\hat{\kappa}(p)=\int_{-\infty}^{\infty} e^{i p u} \kappa(u) d u, \quad p \in R,
$$

plays an important role in the spectral theory for $K$. Since $\kappa \in L^{1}(R)$, $\hat{\kappa}$ is continuous and $\hat{\kappa}(p) \rightarrow 0$ as $p \rightarrow \pm \infty$. The set

$$
\Gamma=\{\hat{\kappa}(p): p \in R\} \cup\{0\}
$$

forms a continuous closed curve in the complex plane C. From Krein [7], $\sigma(K)$ consists of $\Gamma$ and the points $\lambda \in \mathbf{C}$ for which the winding number of $\lambda$ with respect to $\Gamma$ is nonzero.

In this paper we consider the operators $K$ and $K_{\beta}$ acting on $L^{2}\left(R^{+}\right)$ in place of $X^{+}$. The spectra are unchanged. For $\sigma(K)$ this was proved by Krein [7]. For $\sigma\left(K_{\beta}\right)$ it is proved in Section 2 below. The advantage of $L^{2}\left(R^{+}\right)$is that Hilbert space properties and results can be used. Section 2 concludes with a brief discussion of an example for which the spectrum of $K_{\beta}$ is neither trivial nor asymptotically dense in $\sigma(K)$.

It is shown in Section 3 that $K$ and $K_{\beta}$ have certain asymptotic properties which are reminiscent of properties of self-adjoint operators. Let

$$
\varphi_{\beta p}(t)=\left\{\begin{array}{ll}
\frac{1}{\sqrt{\beta}} e^{-i p t}, & 0 \leq t \leq \beta, \\
0, & \beta<t<\infty,
\end{array} \quad \beta \in R^{+}, p \in R .\right.
$$

Then $\varphi_{\beta p} \in L^{2}\left(R^{+}\right),\left\|\varphi_{\beta p}\right\|_{2}=1$, and

$$
\left(\varphi_{\beta p}, \varphi_{\beta q}\right) \rightarrow 0 \quad \text { as } \quad \beta \rightarrow \infty \quad \text { for } p \neq q \text {. }
$$


We prove that

$$
\begin{aligned}
& \left\|\hat{\kappa}(p) \varphi_{\beta p}-K \varphi_{\beta p}\right\|_{2} \rightarrow 0 \quad \text { as } \quad \beta \rightarrow \infty, \quad p \in R, \\
& \left\|\hat{\kappa}(p) \varphi_{\beta p}-K_{\beta} \varphi_{\beta p}\right\|_{2} \rightarrow 0 \quad \text { as } \quad \beta \rightarrow \infty, \quad p \in R .
\end{aligned}
$$

In other words, each $\hat{\kappa}(p)$ is an asymptotic eigenvalue of $K$ and of $K_{\beta}$ as $\beta \rightarrow \infty$, with asymptotic eigenfunctions $\varphi_{\beta p}$. This result for $K$ gives a direct argument, without analytic function theory, that $\hat{\kappa}(p) \in \sigma(K)$ for $p \in R$.

In Section 4 it is assumed that $\kappa \in L^{1}(R)$ and $\kappa(-u)=\overline{\kappa(u)}$. Then $K$ and $K_{\beta}$ are self-adjoint and the spectra $\sigma(K)$ and $\sigma\left(K_{\beta}\right)$ are real. Let

$$
\begin{array}{rlrl}
m & =\min \sigma(K), & M & =\max \sigma(K), \\
m_{\beta} & =\min \sigma\left(K_{\beta}\right), \quad M_{\beta}=\max \sigma\left(K_{\beta}\right) .
\end{array}
$$

Then

$$
\sigma(K)=[m, M], \quad \sigma\left(K_{\beta}\right) \subset\left[m_{\beta}, M_{\beta}\right] .
$$

Moreover, $m \leq 0 \leq M$ and $m<M$. We prove that

$$
\begin{gathered}
{\left[m_{\beta}, M_{\beta}\right] \subset[m, M],} \\
m_{\beta} \rightarrow m \text { and } M_{\beta} \rightarrow M \quad \text { as } \beta \rightarrow \infty, \\
\# \sigma\left(K_{\beta}\right) \rightarrow \infty \text { as } \beta \rightarrow \infty,
\end{gathered}
$$

where $\# \sigma\left(K_{\beta}\right)$, which may be finite or infinite, is the number of nonzero eigenvalues of $K_{\beta}$. Also, we show that the number of eigenvalues of $K_{\beta}$ in any neighborhood of $M$ is unbounded if $M>0$, and we give a lower bound for the sum of the positive eigenvalues of $K_{\beta}$.

Finally, in Section 5, it is proved under more restrictive assumptions on the kernel function $\kappa$ that $\sigma\left(K_{\beta}\right)$ is asymptotically dense in $\sigma(K)$ as $\beta \rightarrow \infty$. The analysis is based on results from the book on Toeplitz forms by Grenander and Szegö [4].

The convolution inequality (see [10])

$$
\begin{array}{cl}
\kappa \in L^{1}(R), \quad g \in L^{2}(R), \quad h(s) & =\int_{-\infty}^{\infty} \kappa(s-t) g(t) d t \\
\Rightarrow h \in L^{2}(R) & \text { and } \quad\|h\|_{2} \leq\|\kappa\|_{1}\|g\|_{2}
\end{array}
$$


will be used on several occasions.

2. General properties of $K$ and $K_{\beta}$. For convenience, the definitions of $K$ and $K_{\beta}$ are repeated:

$$
\begin{aligned}
& K f(s)=\int_{0}^{\infty} \kappa(s-t) f(t) d t, \\
& K_{\beta} f(s)=\int_{0}^{\beta} \kappa(s-t) f(t) d t,
\end{aligned}
$$

where $\kappa \in L^{1}(R)$. By standard arguments (see [2])

$$
K: X^{+} \rightarrow X^{+} \quad \text { with } \quad\|K\|=\|\kappa\|_{1} .
$$

The convolution inequality yields

$$
K: L^{2}\left(R^{+}\right) \rightarrow L^{2}\left(R^{+}\right) \quad \text { with } \quad\|K\| \leq\|\kappa\|_{1} .
$$

By similar reasoning,

$$
\begin{aligned}
K_{\beta}: X^{+} & \rightarrow X^{+} \quad \text { with } \quad\left\|K_{\beta}\right\| \leq\|\kappa\|_{L^{1}(-\beta, \infty)}, \\
K_{\beta}: L^{2}\left(R^{+}\right) & \rightarrow L^{2}\left(R^{+}\right) \quad \text { with } \quad\left\|K_{\beta}\right\| \leq\|\kappa\|_{L^{1}(-\beta, \infty)} .
\end{aligned}
$$

It is convenient to consider $K_{\beta}$ also on $C[0, \beta]$ and $L^{2}(0, \beta)$. Then

$$
\begin{gathered}
K_{\beta}: C[0, \beta] \rightarrow C[0, \beta] \quad \text { with } \quad\left\|K_{\beta}\right\| \leq\|\kappa\|_{L^{1}(-\beta, \beta)}, \\
K_{\beta}: L^{2}[0, \beta] \rightarrow L^{2}[0, \beta] \quad \text { with } \quad\left\|K_{\beta}\right\| \leq\|\kappa\|_{L^{1}(-\beta, \beta)} .
\end{gathered}
$$

We show next that the operators $K_{\beta}$ are compact on each of the four spaces $X^{+}, L^{2}\left(R^{+}\right), C[0, \beta], L^{2}(0, \beta)$. For $K_{\beta}$ on $X^{+}$or $C[0, \beta]$, $\left\{K_{\beta} f:\|f\| \leq 1\right\}$ is bounded and equicontinuous. For $K_{\beta}$ on $X^{+}$we also have $K_{\beta} f(s) \rightarrow 0$ as $s \rightarrow \infty$, uniformly for $\|f\| \leq 1$. It follows that $K_{\beta}$ is compact on $X^{+}$and $C[0, \beta]$. For more details, see [2]. Next, consider $K_{\beta}$ on $L^{2}(0, \beta)$. There exist polynomials $\kappa_{\beta n}(u), n=1,2, \ldots$, such that

$$
\left\|\kappa_{\beta n}-\kappa\right\|_{L^{1}(-\beta, \beta)} \rightarrow 0 \quad \text { as } \quad n \rightarrow \infty .
$$


The corresponding operators $K_{\beta n}$ have finite ranks, hence are compact, and $\left\|K_{\beta n}-K_{\beta}\right\| \rightarrow 0$ as $n \rightarrow \infty$. Therefore, $K_{\beta}$ is compact on $L^{2}(0, \beta)$. Finally, consider $K_{\beta}$ on $L^{2}\left(R^{+}\right)$. Let

$$
\kappa_{m}(u)= \begin{cases}\kappa(u), & u \leq m, \\ 0, & u>m .\end{cases}
$$

Then $\left\|\kappa_{m}-\kappa\right\|_{1} \rightarrow 0$ as $m \rightarrow \infty$. The corresponding operators $K_{\beta m}$ are compact, by an argument like the one given for $K_{\beta}$ on $L^{2}(0, \beta)$, and $\left\|K_{\beta m}-K_{\beta}\right\| \rightarrow 0$ as $m \rightarrow \infty$. Hence, $K_{\beta}$ is compact on $L^{2}\left(R^{+}\right)$.

We prove next that the spectrum of $K_{\beta}$ is the same for $K_{\beta}$ acting on the four spaces. In all four cases, $K_{\beta}$ is a compact operator on an infinite dimensional space, so that 0 is in the spectrum of $K_{\beta}$, and every nonzero element $\lambda$ in the spectrum is an eigenvalue of $K_{\beta}$ with a finite dimensional eigenmanifold $N\left(\lambda I-K_{\beta}\right)$.

Fix $\lambda \neq 0$. The associated eigenvalue problems for $K_{\beta}$ on $X^{+}$and $C[0, \beta]$ are related by

$$
\begin{gathered}
K_{\beta} f=\lambda f, \quad f \in X^{+}, f \neq 0 \\
\Leftrightarrow \\
K_{\beta} f_{\beta}=\lambda f_{\beta}, \quad f_{\beta} \in C[0, \beta], f_{\beta} \neq 0,
\end{gathered}
$$

where $f_{\beta}$ is the restriction of $f$ to $[0, \beta]$ and

$$
f(s)=\frac{1}{\lambda} \int_{0}^{\beta} \kappa(s-t) f_{\beta}(t) d t, \quad s \in R^{+} .
$$

The eigenvalue problems for $K_{\beta}$ on $L^{2}\left(R^{+}\right)$and $L^{2}(0, \beta)$ are related in the same way. The convolution inequality ensures that $f \in L^{2}\left(R^{+}\right)$if $f_{\beta} \in L^{2}(0, \beta)$. These observations imply that

$$
\sigma\left(K_{\beta}\right)_{X^{+}}=\sigma\left(K_{\beta}\right)_{C[0, \beta]}, \quad \sigma\left(K_{\beta}\right)_{L^{2}\left(R^{+}\right)}=\sigma\left(K_{\beta}\right)_{L^{2}(0, \beta)} .
$$

We prove next that

$$
\sigma\left(K_{\beta}\right)_{C[0, \beta]}=\sigma\left(K_{\beta}\right)_{L^{2}(0, \beta)} .
$$

The argument (for which we are indebted to P. Hähner and R. Kress) is based on the theory of dual systems given, for example, in the book by 
Kress [9]. See also Jörgens [5] and Wendland [13]. We summarize the basic ideas. Let $X$ and $Y$ be linear spaces and let $\langle x, y\rangle$ be a bilinear form on $X \times Y$. Assume that $\langle x, y\rangle$ is nondegenerate; that is, for any $x \in X$ there exists $y \in Y$ such that $\langle x, y\rangle \neq 0$, and for any $y \in Y$ there exists $x \in X$ such that $\langle x, y\rangle \neq 0$. The linear spaces $X$ and $Y$ form a dual system $\langle X, Y\rangle$.

Let $X=C[0, \beta]$ and $Y=L^{2}(0, \beta)$. Then $\langle X, Y\rangle$ is a dual system with $\langle f, g\rangle=\int_{0}^{\beta} f(s) g(s) d s, f \in X, g \in Y$, and $\langle Y, Y\rangle$ is a dual system with $\langle f, g\rangle=\int_{0}^{\beta} f(s) g(s) d s, f, g \in Y$. Define $K_{\beta}^{\prime}: Y \rightarrow Y$ by $K_{\beta}^{\prime} g(t)=\int_{0}^{\beta} \kappa(t-s) g(s) d s, g \in Y$. Then $\left\langle K_{\beta} f, g\right\rangle=\left\langle f, K_{\beta}^{\prime} g\right\rangle$ for $f \in X, g \in Y$ and for $f, g \in Y$. Hence, $K_{\beta}^{\prime}$ is the adjoint of $K_{\beta}$ with respect to both of the dual systems $\langle X, Y\rangle$ and $\langle Y, Y\rangle$. For $\lambda \neq 0$, two applications of the Fredholm alternative for dual systems $([\mathbf{9}, \mathrm{Thm}$. 4.17]) yield

$$
\operatorname{dim} N\left(\lambda I-K_{\beta}\right)_{X}=\operatorname{dim} N\left(\lambda I-K_{\beta}^{\prime}\right)_{Y}=\operatorname{dim} N\left(\lambda I-K_{\beta}\right)_{Y} .
$$

Since $X=C[0, \beta]$ and $Y=L^{2}(0, \beta)$, we have established

$$
\operatorname{dim} N\left(\lambda I-K_{\beta}\right)_{C[0, \beta]}=\operatorname{dim} N\left(\lambda I-K_{\beta}\right)_{L^{2}(0, \beta)} .
$$

Since $C[0, \beta] \subset L^{2}(0, \beta)$ as sets,

$$
N\left(\lambda I-K_{\beta}\right)_{C[0, \beta]} \subset N\left(\lambda I-K_{\beta}\right)_{L^{2}(0, \beta)} .
$$

Therefore, the equality of the dimensions implies

$$
N\left(\lambda I-K_{\beta}\right)_{C[0, \beta]}=N\left(\lambda I-K_{\beta}\right)_{L^{2}(0, \beta)}
$$

and

$$
\sigma\left(K_{\beta}\right)_{C[0, \beta]}=\sigma\left(K_{\beta}\right)_{L^{2}(0, \beta)} .
$$

It follows that the spectrum of $K_{\beta}$ is the same for $K_{\beta}$ on the four spaces. Denote the common spectrum by $\sigma\left(K_{\beta}\right)$. Thus,

$$
\sigma\left(K_{\beta}\right)=\sigma\left(K_{\beta}\right)_{X^{+}}=\sigma\left(K_{\beta}\right)_{L^{2}\left(R^{+}\right)}=\sigma\left(K_{\beta}\right)_{C[0, \beta]}=\sigma\left(K_{\beta}\right)_{L^{2}\left(R^{+}\right)} .
$$

Another argument for the equivalence of the spectra, called to our attention by the referee, is based on the following principle. Let $X$ and 
$Y$ be Banach spaces with $Y$ dense in $X$. If $A$ is a Fredholm operator with the same index on $X$ and $Y$, then the null spaces of $A$ in $X$ and $Y$ coincide.

The forgoing argument also shows that, for $\lambda \neq 0$, the associated eigenvalue problems for $K_{\beta}$ on the four spaces are essentially equivalent. In particular,

$$
N\left(\lambda I-K_{\beta}\right)_{X^{+}}=N\left(\lambda I-K_{\beta}\right)_{L^{2}\left(R^{+}\right)} .
$$

Henceforth, assume that $K$ acts on $L^{2}\left(R^{+}\right)$, and that $K_{\beta}$ acts on $L^{2}\left(R^{+}\right)$or $L^{2}(0, \beta)$. The conclusions for $K_{\beta}$ are equally valid in both cases.

We end this section with an example which illustrates known results and which perhaps is rich enough to suggest some new ones.

Example 2.1. Let

$$
\kappa(u)= \begin{cases}e^{u}, & u<0 \\ 2 e^{-u}, & u>0 .\end{cases}
$$

The corresponding Wiener-Hopf operator is given by

$$
K f(s)=2 e^{-s} \int_{0}^{s} e^{t} f(t) d t+e^{s} \int_{s}^{\infty} e^{-t} f(t) d t .
$$

The Fourier transform of $\kappa$ is

$$
\hat{\kappa}(p)=\frac{3+i p}{1+p^{2}}, \quad-\infty<p<\infty .
$$

Write $\hat{\kappa}=x+i y$ to show that the curve $\Gamma=\{\hat{\kappa}(p)\} \cup\{0\}$ is the ellipse

$$
\frac{(x-3 / 2)^{2}}{(3 / 2)^{2}}+\frac{y^{2}}{(1 / 2)^{2}}=1
$$

with center $(3 / 2,0)$, major axis $0 \leq x \leq 3, y=0$, and minor axis $-1 / 2 \leq y \leq 1 / 2, x=3 / 2$. As $p$ increases, $\Gamma$ is traced out in the positive direction. The spectrum of $K$ consists of all points $\lambda$ on or inside this ellipse. 
For this example, the operator $K_{\beta}$ is given by

$$
\begin{gathered}
K_{\beta} f(s)=2 e^{-s} \int_{0}^{s} e^{t} f(t) d t+e^{s} \int_{s}^{\beta} e^{-t} f(t) d t, \quad 0 \leq s \leq \beta, \\
K_{\beta} f(s)=2 e^{-s} \int_{0}^{\beta} e^{t} f(t) d t, \quad s \geq \beta .
\end{gathered}
$$

The spectrum of $K_{\beta}$ consists of 0 and a point spectrum that may be studied, for example, by observing that the eigenvalue problem $K_{\beta} f=\lambda f$ is equivalent to a two-point boundary value problem

$$
\begin{gathered}
\lambda f^{\prime \prime}(s)-f^{\prime}(s)+(3-\lambda) f(s)=0, \quad 0 \leq s \leq \beta, \\
\lambda f^{\prime}(0)=(1-\lambda) f(0), \quad \lambda f^{\prime}(\beta)=(1+\lambda) f(\beta) .
\end{gathered}
$$

We omit the technical details (which in fact are not easy), and merely report the salient features of the spectrum of $K_{\beta}$.

The eigenvalues of $K_{\beta}$ either lie on the real interval $(0,3 / 2+\sqrt{2})$ or occur as complex conjugate pairs inside a circle of radius $1 / 12$ centered at $(1 / 12,0)$. The eigenvalue with maximum absolute value is real, simple, and has a corresponding positive eigenfunction. This may be seen as a consequence of the Perron theory, as generalized from positive matrices to an abstract setting which includes integral operators with nonnegative kernels by Krein and Rutman [8]. As $\beta$ increases from 0 , that largest eigenvalue increases monotonically from 0 and approaches $3 / 2+\sqrt{2}$ as $\beta \rightarrow \infty$. All other eigenvalues follow trajectories lying off the real axis for small values of $\beta$, with complex conjugate pairs eventually meeting at a point in the open interval $(3 / 2-\sqrt{2}, 3 / 2+\sqrt{2})$, then forming a real pair, one of which approaches $3 / 2-\sqrt{2}$, and the other approaches $3 / 2+\sqrt{2}$ as $\beta \rightarrow \infty$.

As $\beta \rightarrow \infty$, the spectrum of $K_{\beta}$ becomes asymptotically dense in the interval $[3 / 2-\sqrt{2}, 3 / 2+\sqrt{2}]$. Asymptotic density is believed to occur also for the circle of radius $1 / 12$ and center $(0,1 / 12)$. On the other hand, the complex plane outside this circle and outside the interval $[3 / 2-\sqrt{2}, 3 / 2+\sqrt{2}]$ remains totally unpopulated by eigenvalues of $K_{\beta}$ for any $\beta$. Therefore, $\sigma\left(K_{\beta}\right)$ is not asymptotically dense in $\sigma(K)$ as $\beta \rightarrow \infty$. Present theories, including that which follows, seem inadequate to explain the richness of the behavior of $\sigma\left(K_{\beta}\right)$. 
3. Asymptotic spectral properties of $K$ and $K_{\beta}$. The operators $K$ and $K_{\beta}$ have certain asymptotic properties which are reminiscent of properties of self-adjoint operators. The curve $\Gamma=\{\hat{\kappa}(p)\} \cup\{0\}$ takes on the role of the real spectrum of a self-adjoint operator. The functions

$$
\varphi_{\beta p}(t)= \begin{cases}\frac{1}{\sqrt{\beta}} e^{-i p t}, & 0 \leq t \leq \beta, \\ 0, & \beta<t<\infty,\end{cases}
$$

behave asymptotically like orthonormal eigenfunctions. By easy calculations, $\varphi_{\beta p} \in L^{2}\left(R^{+}\right),\left\|\varphi_{\beta p}\right\|_{2}=1$, and $\left(\varphi_{\beta p}, \varphi_{\beta q}\right) \rightarrow 0$ as $\beta \rightarrow \infty$ for $p \neq q$, uniformly for $|p-q|>\delta$ with any $\delta>0$.

The following theorem shows that, for each $p \in R, \hat{\kappa}(p)$ is an asymptotic eigenvalue of $K$ with asymptotic eigenfunctions $\varphi_{\beta p}$.

\section{Theorem 3.1.}

(a) $\left\|\hat{\kappa}(p) \varphi_{\beta p}-K \varphi_{\beta p}\right\|_{2} \rightarrow 0$ as $\beta \rightarrow \infty$, uniformly for $p \in R$.

(b) $\left(K \varphi_{\beta p}, \varphi_{\beta p}\right) \rightarrow \hat{\kappa}(p)$ as $\beta \rightarrow \infty$, uniformly for $p \in R$.

Proof. Since (a) implies (b), it suffices to prove (a). Since $\kappa \in L^{1}(R)$, for each $\varepsilon>0$ there exists $\alpha=\alpha(\varepsilon)>0$ such that

$$
\left(\int_{-\infty}^{-\alpha}+\int_{\alpha}^{\infty}\right)|\kappa(u)| d u<\varepsilon
$$

This will be used at two places in our analysis. Note that

$$
K \varphi_{\beta p}(s)=\frac{1}{\sqrt{\beta}} \int_{0}^{\beta} \kappa(s-t) e^{-i p t} d t=\frac{e^{-i p s}}{\sqrt{\beta}} \int_{s-\beta}^{s} \kappa(u) e^{i p u} d u
$$

Therefore, for $0 \leq s \leq \beta$,

$$
\begin{gathered}
\hat{\kappa}(p) \varphi_{\beta p}(s)-K \varphi_{\beta p}(s)=\frac{e^{-i p s}}{\sqrt{\beta}}\left(\int_{-\infty}^{s-\beta}+\int_{s}^{\infty}\right) \kappa(u) e^{i p u} d u \\
\left|\hat{\kappa}(p) \varphi_{\beta p}(s)-K \varphi_{\beta p}(s)\right| \leq \frac{1}{\sqrt{\beta}}\left(\int_{-\infty}^{s-\beta}+\int_{s}^{\infty}\right)|\kappa(u)| d u \leq \frac{1}{\sqrt{\beta}}\|\kappa\|_{1} .
\end{gathered}
$$


Fix $\varepsilon>0$. Let $\beta>2 \alpha$, where $\alpha=\alpha(\varepsilon)$. Then $0<\alpha<\beta-\alpha<\beta$ and

$$
\begin{aligned}
\left\|\hat{\kappa}(p) \varphi_{\beta p}-K \varphi_{\beta p}\right\|_{L^{2}(0, \alpha)}^{2} & \leq \frac{\alpha}{\beta}\|\kappa\|_{1}^{2}, \\
\left\|\hat{\kappa}(p) \varphi_{\beta p}-K \varphi_{\beta p}\right\|_{L^{2}(\alpha, \beta-\alpha)}^{2} & \leq \frac{\beta-2 \alpha}{\beta} \varepsilon^{2}, \\
\left\|\hat{\kappa}(p) \varphi_{\beta p}-K \varphi_{\beta p}\right\|_{L^{2}(\beta-\alpha, \beta)}^{2} & \leq \frac{\alpha}{\beta}\|\kappa\|_{1}^{2} .
\end{aligned}
$$

It follows that

$$
\begin{gathered}
\left\|\hat{\kappa}(p) \varphi_{\beta p}-K \varphi_{\beta p}\right\|_{L^{2}(0, \beta)}^{2} \leq \frac{2 \alpha}{\beta}\|\kappa\|_{1}^{2}+\varepsilon^{2}, \\
\left\|\hat{\kappa}(p) \varphi_{\beta p}-K \varphi_{\beta p}\right\|_{L^{2}(0, \beta)} \rightarrow 0 \quad \text { as } \quad \beta \rightarrow \infty, \quad \text { uniformly for } p \in R .
\end{gathered}
$$

It may be remarked that the last result is already enough to yield (b).

To complete the proof of (a), it remains to show that

$\left\|\hat{\kappa}(p) \varphi_{\beta p}-K \varphi_{\beta p}\right\|_{L^{2}(\beta, \infty)} \rightarrow 0 \quad$ as $\quad \beta \rightarrow \infty, \quad$ uniformly for $p \in R$.

Since $\varphi_{\beta p}(s)=0$ for $s>\beta$, this is equivalent to

$$
\left\|K \varphi_{\beta p}\right\|_{L^{2}(\beta, \infty)} \rightarrow 0 \quad \text { as } \quad \beta \rightarrow \infty, \quad \text { uniformly for } p \in R .
$$

First,

$$
\left|K \varphi_{\beta p}(s)\right| \leq \frac{1}{\sqrt{\beta}} \int_{s-\beta}^{s}|\kappa(u)| d u .
$$

Then

$$
\begin{aligned}
&\left\|K \varphi_{\beta p}\right\|_{L^{2}(\beta, \infty)}^{2} \leq \frac{1}{\beta} \int_{\beta}^{\infty}\left(\int_{s-\beta}^{s}|\kappa(u)| d u\right)^{2} d s \\
&=\frac{1}{\beta} \int_{\beta}^{\infty}\left(\int_{s-\beta}^{s}|\kappa(u)| d u\right)\left(\int_{s-\beta}^{s}|\kappa(v)| d v\right) d s \\
&=\frac{1}{\beta} \int_{\beta}^{\infty}\left(\int_{0}^{\infty}|\kappa(u)| \chi_{[s-\beta, s]}(u) d u\right) \\
& \cdot\left(\int_{0}^{\infty}|\kappa(v)| \chi_{[s-\beta, s]}(v) d v\right) d s \\
&=\frac{1}{\beta} \int_{0}^{\infty}|\kappa(u)| \int_{0}^{\infty}|\kappa(v)| \\
& \cdot \int_{\beta}^{\infty} \chi_{[s-\beta, s]}(u) \chi_{[s-\beta, s]}(v) d s d v d u
\end{aligned}
$$


Let

$$
F_{\beta}(u, v)=\int_{\beta}^{\infty} \chi_{[s-\beta, s]}(u) \chi_{[s-\beta, s]}(v) d s .
$$

Then

$$
\left\|K \varphi_{\beta p}\right\|_{L^{2}(\beta, \infty)}^{2} \leq \frac{1}{\beta} \int_{0}^{\infty}|\kappa(u)| \int_{0}^{\infty}|\kappa(v)| F_{\beta}(u, v) d v d u
$$

Since $F_{\beta}(u, v)=F_{\beta}(v, u)$, the integrand is symmetric in $u$ and $v$. So the integrals with $u \leq v$ and $v \leq u$ are equal, and

$$
\left\|K \varphi_{\beta p}\right\|_{L^{2}(\beta, \infty)}^{2} \leq \frac{2}{\beta} \int_{0}^{\infty}|\kappa(u)| \int_{u}^{\infty}|\kappa(v)| F_{\beta}(u, v) d v d u
$$

Consider $F_{\beta}(u, v)$ for $u \leq v$. Note that

$$
\begin{gathered}
\chi_{[s-\beta, s]}(u)=\chi_{[u, u+\beta]}(s), \quad \chi_{[s-\beta, s]}(v)=\chi_{[v, v+\beta]}(s), \\
\chi_{[s-\beta, s]}(u) \chi_{[s-\beta, s]}(v)= \begin{cases}\chi_{[v, u+\beta]}(s) & \text { if } u \leq v \leq u+\beta, \\
0 & \text { if } v>u+\beta .\end{cases}
\end{gathered}
$$

Hence,

$$
\begin{gathered}
F_{\beta}(u, v)=\int_{\beta}^{\infty} \chi_{[v, u+\beta]}(s) d s \quad \text { if } u \leq v \leq u+\beta, \\
F_{\beta}(u, v)=0 \quad \text { if } v>u+\beta .
\end{gathered}
$$

The integral for $F_{\beta}(u, v)$ reduces to

$$
\begin{gathered}
F_{\beta}(u, v)=u \quad \text { if } u \leq v \leq \beta, \\
F_{\beta}(u, v)=u+\beta-v \quad \text { if } \beta \leq v \leq u+\beta .
\end{gathered}
$$


It follows that

$$
\begin{aligned}
\left\|K \varphi_{\beta p}\right\|_{L^{2}(\beta, \infty)}^{2} \leq & \frac{2}{\beta} \int_{0}^{\beta}|\kappa(u)| \int_{u}^{\beta}|\kappa(v)| u d v d u \\
& +\frac{2}{\beta} \int_{0}^{\beta}|\kappa(u)| \int_{\beta}^{u+\beta}|\kappa(v)|(u+\beta-v) d v d u \\
& +\frac{2}{\beta} \int_{\beta}^{\infty}|\kappa(u)| \int_{u}^{u+\beta}|\kappa(v)|(u+\beta-v) d v d u, \\
\left\|K \varphi_{\beta p}\right\|_{L^{2}(\beta, \infty)}^{2} \leq & \frac{2}{\beta} \int_{0}^{\beta}|\kappa(u)| u \int_{u}^{u+\beta}|\kappa(v)| d v d u \\
& +2 \int_{\beta}^{\infty}|\kappa(u)| \int_{u}^{u+\beta}|\kappa(v)| d v d u, \\
\left\|K \varphi_{\beta p}\right\|_{L^{2}(\beta, \infty)}^{2} \leq & 2\|\kappa\|_{1}\left\{\frac{1}{\beta} \int_{0}^{\beta}|\kappa(u)| u d u+\int_{\beta}^{\infty}|\kappa(u)| d u\right\} .
\end{aligned}
$$

For $\beta>\alpha=\alpha(\varepsilon)$,

$$
\begin{aligned}
\frac{1}{\beta} \int_{0}^{\beta}|\kappa(u)| u d u & \leq \frac{\alpha}{\beta} \int_{0}^{\alpha}|\kappa(u)| d u+\int_{\alpha}^{\beta}|\kappa(u)| d u \\
& \leq \frac{\alpha}{\beta}\|\kappa\|_{1}+\varepsilon .
\end{aligned}
$$

Hence,

$$
\frac{1}{\beta} \int_{0}^{\beta}|\kappa(u)| u d u \rightarrow 0 \quad \text { as } \quad \beta \rightarrow \infty
$$

It follows that

$$
\left\|K \varphi_{\beta p}\right\|_{L^{2}(\beta, \infty)}^{2} \rightarrow 0 \quad \text { as } \quad \beta \rightarrow \infty, \quad \text { uniformly for } p \in R,
$$

which completes the proof. $\quad \square$

Next, consider the operators $K_{\beta}$ on $L^{2}\left(R^{+}\right)$. Since $\varphi_{\beta p}(s)=0$ for $s>\beta, K_{\beta} \varphi_{\beta p}=K \varphi_{\beta p}$. An immediate consequence of Theorem 3.1 is

\section{Theorem 3.2.}

(a) $\left\|\hat{\kappa}(p) \varphi_{\beta p}-K_{\beta} \varphi_{\beta p}\right\|_{2} \rightarrow 0$ as $\beta \rightarrow \infty$, uniformly for $p \in R$. 
(b) $\left(K_{\beta} \varphi_{\beta p}, \varphi_{\beta p}\right) \rightarrow \hat{\kappa}(p)$ as $\beta \rightarrow \infty$, uniformly for $p \in R$.

Thus, for each $p \in R, \hat{\kappa}(p)$ is an asymptotic eigenvalue of $K_{\beta}$ as $\beta \rightarrow \infty$, with asymptotic eigenfunctions $\varphi_{\beta p}$. The forgoing conclusions remain valid if the setting is $L^{2}(0, \beta)$ instead of $L^{2}\left(R^{+}\right)$.

Example 3.1. Let $p_{j}=p_{\beta j}=2 \pi j / \beta$ for $j \in Z$. Then $\left(\varphi_{\beta p_{j}}, \varphi_{\beta p_{k}}\right)=$ $\delta_{j k}$. Since $\hat{\kappa}$ is uniformly continuous, it follows from Theorem 3.2 that $\left\{\left(K_{\beta} \varphi_{\beta p_{j}}, \varphi_{\beta p_{j}}\right): j \in Z\right\}$ is asymptotically dense in $\Gamma=\{\hat{\kappa}(p)\} \cup\{0\}$ as $\beta \rightarrow \infty$. It does not matter whether the setting is $L^{2}\left(R^{+}\right)$or $L^{2}(0, \beta)$.

4. Self-adjoint operators. Throughout this section, assume that

$$
\kappa \in L^{1}(R), \quad\|\kappa\|_{1} \neq 0, \quad \kappa(-u)=\overline{\kappa(u)} .
$$

Then $K \neq 0$ and $K$ is self-adjoint on $L^{2}\left(R^{+}\right)$. The spectrum of $K$ is a real interval: $\sigma(K)=\{\hat{\kappa}(p)\} \cup\{0\}=[m, M]$, where $m \leq 0 \leq M$ and $m<M$. Thus, $M>0$ or $m<0$ or both. If $M>0$, then $M=\hat{\kappa}\left(p_{0}\right)$ for some $p_{0} \in R$ and similarly if $m<0$.

The operators $K_{\beta}$ are compact and self-adjoint on $L^{2}\left(R^{+}\right)$and on $L^{2}(0, \beta)$. The spectrum of $K_{\beta}$ is the same in both cases. From now on, unless otherwise indicated, we shall assume that $K_{\beta}$ acts on $L^{2}(0, \beta)$. For simplicity, denote

$$
\|f\|=\|f\|_{L^{2}(0, \beta)} \quad \text { for } f \in L^{2}(0, \beta) .
$$

Let

$$
M_{\beta}=\max \sigma\left(K_{\beta}\right), \quad m_{\beta}=\min \sigma\left(K_{\beta}\right) .
$$

Then

$$
\sigma\left(K_{\beta}\right) \subset\left[m_{\beta}, M_{\beta}\right], \quad m_{\beta} \leq 0 \leq M_{\beta} .
$$

Furthermore,

$$
M_{\beta}=\sup _{\|f\|=1}\left(K_{\beta} f, f\right), \quad m_{\beta}=\inf _{\|f\|=1}\left(K_{\beta} f, f\right) .
$$

If $M_{\beta}>0$, then $M_{\beta}$ is the maximum of the quadratic form and $M_{\beta}$ is the largest eigenvalue of $K_{\beta}$. Similarly, if $m_{\beta}<0$, then $m_{\beta}$ is the minimum of the quadratic form and $m_{\beta}$ is the smallest eigenvalue of $K_{\beta}$. 
Example 4.1. Let $\kappa(u)=e^{-|u|}$, the Picard kernel. Then

$$
\hat{k}(p)=\frac{2}{1+p^{2}}, \quad \sigma(K)=[0,2],
$$

and $\lambda \in(0,2)$ is an eigenvalue of $K_{\beta}$ if and only if

$$
\lambda=\frac{2}{\gamma^{2}+1}, \quad \tan \beta \gamma=\frac{2 \gamma}{\gamma^{2}-1}, \quad \gamma>0 .
$$

Thus, $\sigma\left(K_{\beta}\right) \subset \sigma(K)$. By a graphical argument, there is at least one solution $\gamma$ in almost every interval of length $\pi / \beta$, so that $\sigma\left(K_{\beta}\right)$ is asymptotically dense in $\sigma(K)$ as $\beta \rightarrow \infty$. Hence,

$$
M_{\beta} \rightarrow M \quad \text { and } \quad m_{\beta} \rightarrow m \quad \text { as } \quad n \rightarrow \infty .
$$

See [2] for further details. The analysis is given there for $K$ and $K_{\beta}$ acting on $X^{+}$. However, $\sigma(K)$ and $\sigma\left(K_{\beta}\right)$ are the same as in the present situation.

Conjecture. If $\kappa \in L^{1}(R),\|\kappa\|_{1} \neq 0$, and $\kappa(-u)=\overline{\kappa(u)}$, then $\sigma\left(K_{\beta}\right)$ is asymptotically dense in $\sigma(K)$ as $\beta \rightarrow \infty$.

We shall prove in the next section that the asymptotic density is obtained under the additional condition $\hat{\kappa} \in L^{1}(R)$, which is satisfied by Example 4.1. While the issue is not resolved for more general kernel functions $\kappa$, some results in this direction are established below. The number of eigenvalues of $K_{\beta}$, counting multiplicities, tends to infinity as $\beta \rightarrow \infty$. Lower bounds are derived for leading eigenvalues and for sums of eigenvalues of $K_{\beta}$.

The following notation will be needed. Let

$$
\sigma^{+}\left(K_{\beta}\right)=\left\{\lambda \in \sigma\left(K_{\beta}\right): \lambda>0\right\}, \quad \sigma^{-}\left(K_{\beta}\right)=\left\{\lambda \in \sigma\left(K_{\beta}\right): \lambda<0\right\} .
$$

Whenever these sets are nonvoid, let

$$
\sigma^{+}\left(K_{\beta}\right)=\left\{\lambda_{\beta j}: j=1,2, \ldots\right\}, \quad \sigma^{-}\left(K_{\beta}\right)=\left\{\mu_{\beta j}: j=1,2, \ldots\right\},
$$

where the eigenvalues $\lambda_{\beta k}$ and $\mu_{\beta k}$ are repeated as to multiplicity, and

$$
M_{\beta}=\lambda_{\beta 1} \geq \lambda_{\beta 2} \geq \cdots>0, \quad m_{\beta}=\mu_{\beta 1} \leq \mu_{\beta 2} \leq \cdots<0 .
$$


The numbers of eigenvalues in $\sigma^{+}\left(K_{\beta}\right)$ and $\sigma^{-}\left(K_{\beta}\right)$ are denoted by $\# \sigma^{+}\left(K_{\beta}\right)$ and $\# \sigma^{-}\left(K_{\beta}\right)$, either finite or infinite. If $\# \sigma^{+}\left(K_{\beta}\right)=\infty$, then $\lambda_{\beta j} \rightarrow 0$ as $j \rightarrow \infty$, since $K_{\beta}$ is compact. Similarly, $\mu_{\beta j} \rightarrow 0$ as $j \rightarrow \infty$ if $\# \sigma^{-}\left(K_{\beta}\right)=\infty$. Finally, the number of nonzero eigenvalues in $\sigma\left(K_{\beta}\right)$ is

$$
\# \sigma\left(K_{\beta}\right)=\# \sigma^{+}\left(K_{\beta}\right)+\# \sigma^{-}\left(K_{\beta}\right) .
$$

Results will be proved primarily for $\sigma^{+}\left(K_{\beta}\right)$. They carry over to $\sigma^{-}\left(K_{\beta}\right)$ by replacing $\kappa(u)$ by $-\kappa(u)$.

Theorem 4.1. Assume $\kappa \in L^{1}(R),\|\kappa\|_{1} \neq 0$, and $\kappa(-u)=\overline{\kappa(u)}$. Let $0<\alpha<\beta<\infty$. Then

(a) $\# \sigma^{ \pm}\left(K_{\alpha}\right) \leq \# \sigma^{ \pm}\left(K_{\beta}\right), \# \sigma\left(K_{\alpha}\right) \leq \# \sigma\left(K_{\beta}\right)$,

(b) $\lambda_{\alpha j} \leq \lambda_{\beta j}$ for $j \leq \# \sigma^{+}\left(K_{\alpha}\right), \mu_{\alpha j} \geq \mu_{\beta j}$ for $j \leq \# \sigma^{-}\left(K_{\alpha}\right)$.

(c) $\left[m_{\alpha}, M_{\alpha}\right] \subset\left[m_{\beta}, M_{\beta}\right]$.

Proof. Let $V$ be the closed subspace of $L^{2}(0, \beta)$ defined by

$$
V=\left\{f \in L^{2}(0, \beta): f(s)=0 \text { for } \alpha<s<\beta\right\} .
$$

The monotonicity theorem associated with the Rayleigh-Ritz method (see Theorem A1 in the Appendix) states that the positive eigenvalues of the quadratic form $\left(K_{\beta} f, f\right)$ on $V$, when appropriately labelled, are no greater than those of $\left(K_{\beta} f, f\right)$ on $L^{2}(0, \beta)$. Since

$$
\left(K_{\beta} f, f\right)=\left(K_{\alpha} f, f\right)_{[0, \alpha]} \quad \text { for } f \in V,
$$

the result translates as

$$
\lambda_{\alpha j} \leq \lambda_{\beta j} \quad \text { for } j \leq \# \sigma^{+}\left(K_{\alpha}\right) .
$$

A similar argument for the negative eigenvalues yields (a) and (b). Set $j=1$ in (b) to obtain (c).

Theorem 4.2. Let $\kappa \in L^{1}(R),\|\kappa\|_{1} \neq 0$, and $\kappa(-u)=\overline{\kappa(u)}$.

(a) $\sigma\left(K_{\beta}\right) \subset\left[m_{\beta}, M_{\beta}\right] \subset[m, M]=\sigma(K)$ for all $\beta$.

(b) $M_{\beta} \rightarrow M$ and $m_{\beta} \rightarrow m$ as $\beta \rightarrow \infty$. 
(c) For $\beta$ sufficiently large, $K_{\beta}$ has at least one nonzero eigenvalue: a positive eigenvalue if $M>0$, and a negative eigenvalue if $m<0$.

Proof. By Theorem 3.15 of [3], every neighborhood of $\sigma(K)=[m, M]$ contains $\sigma\left(K_{\beta}\right)$, and hence, contains $\left[m_{\beta}, M_{\beta}\right]$, for $\beta$ large enough. From Theorem 4.1(c), $M_{\alpha} \leq M_{\beta}$ for $\alpha<\beta$. It follows by contradiction that $M_{\beta} \leq M$ for all $\beta$. If $M=0$, then $M_{\beta}=0$. Suppose $M>0$. Then $\hat{\kappa}\left(p_{0}\right)=M$ for some $p_{0} \in R$. By Theorem 3.2,

$$
\left(K_{\beta} \varphi_{\beta p_{0}}, \varphi_{\beta p_{0}}\right) \rightarrow \hat{\kappa}\left(p_{0}\right)=M \quad \text { as } \beta \rightarrow \infty .
$$

Since $M_{\beta}=\sup _{\|f\|=1}\left(K_{\beta} f, f\right)$ and $M_{\beta} \leq M, M_{\beta} \rightarrow M$ as $\beta \rightarrow \infty$. The other results follow.

The next theorem shows that $\# \sigma\left(K_{\beta}\right) \rightarrow \infty$ as $\beta \rightarrow \infty$. Moreover, if $M>0$, then the number of eigenvalues of $K_{\beta}$ in any neighborhood of $M$ grows without bound as $\beta \rightarrow \infty$.

Theorem 4.3. Assume $\kappa \in L^{1}(R),\|\kappa\|_{1} \neq 0$, and $\kappa(-u)=\overline{\kappa(u)}$.

(a) $\# \sigma\left(K_{\beta}\right) \rightarrow \infty$ as $\beta \rightarrow \infty$.

(b) If $M>0$, then $\# \sigma^{+}\left(K_{\beta}\right) \rightarrow \infty$ as $\beta \rightarrow \infty$; moreover

$$
\lambda_{\beta j} \rightarrow M \quad \text { as } \beta \rightarrow \infty \quad \text { for } j=1,2, \ldots .
$$

Proof. Let $M>0$. Then $M=\hat{\kappa}\left(p_{0}\right)$ for some $p_{0} \in R$. From Theorem 3.2(b) and Example 3.1, for any $n \geq 1$ there exists $\beta(n)$ such that for each $\beta \geq \beta(n)$ there is an orthonormal set $\left\{x_{\beta j}: j=1, \ldots, n\right\}$ in $L^{2}(0, \beta)$ which satisfies

$$
\left(K_{\beta} x_{\beta j}, x_{\beta j}\right)>\frac{n-1}{n} M \quad \text { for } j=1, \ldots, n .
$$

Since $M \geq M_{\beta}=\lambda_{\beta 1}$, the last inequality and Theorem A2(a) in the Appendix imply that $\# \sigma^{+}\left(K_{\beta}\right) \geq n$ for $\beta \geq \beta(n)$. Therefore, $\# \sigma^{+}\left(K_{\beta}\right) \rightarrow \infty$ as $\beta \rightarrow \infty$. For the remainder of the proof, assume that $\beta \geq \beta(n)$. For $j=1, \ldots, n$,

$$
\lambda_{\beta 1}=M_{\beta} \geq\left(K_{\beta} x_{\beta j}, x_{\beta j}\right)>\frac{n-1}{n} M .
$$


From Theorem A2(b), it follows that

$$
\lambda_{\beta j}>\frac{n-j}{n-j+1} \lambda_{\beta 1} \geq \frac{n-j}{n} \lambda_{\beta 1} \quad \text { for } j=1, \ldots, n .
$$

Hence,

$$
M \geq \lambda_{\beta j}>\frac{n-j}{n} \cdot \frac{n-1}{n} M>\frac{n-j-1}{n} M \quad \text { for } j=1, \ldots, n .
$$

Fix any $j \geq 1$. Let $n \rightarrow \infty$ and $\beta \rightarrow \infty$ with $\beta \geq \beta(n)$, to obtain $\lambda_{\beta j} \rightarrow M$ as $\beta \rightarrow \infty$. Thus, (b) is proved. A similar result holds if $m<0$, and now (a) follows.

The following theorem specifies in further detail how the positive eigenvalues $\lambda_{\beta j}$ of $K_{\beta}$ depend on $\beta$. It augments Theorems 4.1 and 4.3. Among other results, $\lambda_{\beta j}$ is a continuous function of $\beta$ for each $j$. The analysis is based on the collectively compact operator theory in [1]. The results in $[\mathbf{1}]$ are for operator sequences, but they are equally valid with the same proofs for operators depending on a continuous parameter such as $\beta$.

Theorem 4.4. Assume $\kappa \in L^{1}(R), \kappa(-u)=\overline{\kappa(u)}$, and $M>0$.

(a) There is a sequence $\left\{\alpha_{j}\right\}$ such that $0 \leq \alpha_{j} \leq \alpha_{j+1}<\infty$ and such that $\lambda_{\beta j}$ exists if and only if $\beta \in\left(\alpha_{j}, \infty\right\}$. Moreover, $\lambda_{\beta j}$ is a continuous function of $\beta$ for $\beta \in\left(\alpha_{j}, \infty\right)$.

(b) $\left\{\beta: \# \sigma^{+}\left(K_{\beta}\right)=j\right\}=\left(\alpha_{j}, \alpha_{j+1}\right]$.

(c) $\lambda_{\beta j} \rightarrow 0$ as $\beta \rightarrow \alpha_{j}+$.

(d) If $\# \sigma^{+}\left(K_{\beta}\right)=\infty$ for some $\beta$ and if $\# \sigma^{+}\left(K_{\alpha}\right)<\infty$ for $\alpha<\beta$, then $\# \sigma^{+}\left(K_{\alpha}\right) \rightarrow \infty$ as $\alpha \rightarrow \beta-$.

Proof. In this proof, we assume that $K_{\beta}$ acts on $X^{+}$rather than $L^{2}(0, \beta)$. The spectrum of $K_{\beta}$ is unchanged. For $0 \leq \alpha<\beta<\infty$,

$$
\begin{aligned}
\left|K_{\beta} f(s)-K_{\alpha} f(s)\right| & \leq \int_{\alpha}^{\beta}|\kappa(s-t) f(t)| d t \\
& \leq\|f\|_{\infty} \int_{s-\beta}^{s-\alpha}|\kappa(u)| d u
\end{aligned}
$$




$$
\begin{gathered}
\left\|K_{\beta}-K_{\alpha}\right\| \leq \sup _{r \in R} \int_{r}^{r+\beta-\alpha}|\kappa(u)| d u, \\
\left\|K_{\beta}-K_{\alpha}\right\| \rightarrow 0 \quad \text { as } \quad \beta-\alpha \rightarrow 0 .
\end{gathered}
$$

Since each operator $K_{\beta}$ is compact, it follows by a simple argument (see $[\mathbf{1}$, Proposition 5.3]) that

$$
\left\{K_{\beta}: 0 \leq \beta \leq \gamma\right\} \text { is collectively compact for any } \gamma \in R^{+} .
$$

For any $r>0$, let $\# \sigma_{r}\left(K_{\beta}\right)$ be the number of eigenvalues $\lambda_{\beta j}$ of $K_{\beta}$ with $\lambda_{\beta j}>r$ (counting multiplicities). Since $K_{\beta}$ is compact, $\# \sigma_{r}\left(K_{\beta}\right)$ is finite. In other terminology, $\# \sigma_{r}\left(K_{\beta}\right)$ is the dimension of the spectral subspace associated with the spectral set $\left\{\lambda \in \sigma\left(K_{\beta}\right): \lambda>r\right\}$. By the continuous analogue of Theorem 4.16 in [1], there exists $\delta(\beta, r)>0$ such that

$$
\begin{aligned}
& \# \sigma_{r}\left(K_{\alpha}\right)=\# \sigma_{r}\left(K_{\beta}\right) \quad \text { for }|\alpha-\beta|<\delta(\beta, r), \\
& \lambda_{\alpha j} \rightarrow \lambda_{\beta j} \quad \text { as } \quad \alpha \rightarrow \beta \text { for } j \leq \# \sigma_{r}\left(K_{\beta}\right) .
\end{aligned}
$$

Thus, for each $j, \lambda_{\beta j}$ is a continuous function of $\beta$ for $\beta$ in an open set which, by Theorems 4.1 and 4.3 , has the form $\left(\alpha_{j}, \infty\right)$ with $0 \leq \alpha_{j} \leq \alpha_{j+1}<\infty$. This proves (a), which implies (b). Consider (c). First, assume $\alpha_{j}=0$ for some $j$. Let $\beta \rightarrow 0$. Then $M_{\beta} \leq\left\|K_{\beta}\right\| \rightarrow 0$ as $\beta \rightarrow 0$, and (b) is valid for this case. Now consider (c) with $\alpha_{j}>0$. By (b), \# $\sigma^{+}\left(K_{\alpha_{j}}\right)<j$. Since $\# \sigma^{+}\left(K_{\alpha_{j}}\right)$ is finite, there exists $r_{j}>0$ such that

$$
\# \sigma_{r}\left(K_{\alpha j}\right)=\# \sigma^{+}\left(K_{\alpha j}\right)<j \text { for } 0<r<r_{j} .
$$

From above, $\# \sigma_{r}\left(K_{\beta}\right)=\# \sigma_{r}\left(K_{\alpha j}\right)<j$ for $\left|\beta-\alpha_{j}\right|<\delta\left(\alpha_{j}, r\right)$, $0<r<r_{j}$. Since $\lambda_{\beta 1} \geq \lambda_{\beta 2} \geq \cdots \geq \lambda_{\beta j}>0$ for $\beta>\alpha_{j}$, it follows that $\lambda_{\beta j} \leq r$ for $\alpha_{j}<\beta_{j}<\alpha_{j}+\delta\left(\alpha_{j}, r\right), 0<r<r_{j}$, which implies (c). Finally, consider (d). From above, $\# \sigma^{+}\left(K_{\alpha}\right) \geq \# \sigma_{r}\left(K_{\alpha}\right)=\# \sigma_{r}\left(K_{\beta}\right)$ for $\beta-\delta(\beta, r)<\alpha<\beta$. Let $r \rightarrow \infty$ to obtain (d).

The final theorem in this section gives estimates for sums of eigenvalues of $K_{\beta}$. When all the eigenvalues are positive, trace estimates are obtained. In the theorem

$$
\hat{\kappa}_{+}(p)= \begin{cases}\hat{\kappa}(p) & \text { if } \hat{\kappa}(p) \geq 0 \\ 0 & \text { if } \hat{\kappa}(p)<0\end{cases}
$$


Since $\kappa \in L^{1}(R)$, both $\hat{\kappa}(p)$ and $\hat{\kappa}_{+}(p)$ are continuous and approach zero as $p \rightarrow \pm \infty$.

Theorem 4.5. Assume $\kappa \in L^{1}(R),\|\kappa\|_{1} \neq 0$ and $\kappa(-u)=\overline{\kappa(u)}$.

(a) $\liminf _{\beta \rightarrow \infty} \frac{2 \pi}{\beta} \sum_{\lambda \in \sigma^{+}\left(K_{\beta}\right)} \lambda \geq \lim _{P \rightarrow \infty} \int_{-P}^{P} \hat{\kappa}_{+}(p) d p$.

(b) $\liminf _{\beta \rightarrow \infty} \frac{2 \pi}{\beta} \sum_{\lambda \in \sigma\left(K_{\beta}\right)}|\lambda| \geq \lim _{P \rightarrow \infty} \int_{-P}^{P} \hat{\kappa}_{+}(p) d p$.

The limits on the right can be finite or infinite.

Proof. Define $p_{j}=p_{\beta j}=2 \pi j / \beta$ for $j \in Z$. Then, as in Example 3.1, $\left\{\varphi_{\beta p_{j}}: j \in Z\right\}$ is an orthonormal set in $L^{2}(0, \beta)$. So too is any subset, such as the subset for which $\hat{\kappa}\left(p_{j}\right) \geq 0$. It therefore follows from Theorem A3 in the Appendix that, for any $P>0$,

$$
\sum_{\lambda \in \sigma^{+}\left(K_{\beta}\right)} \lambda \geq \sum_{\substack{j \in Z \\ \hat{\kappa}\left(p_{j}\right) \geq 0 \\\left|p_{j}\right| \leq P}}\left(K_{\beta} \varphi_{\beta p_{j}}, \varphi_{\beta p_{j}}\right) .
$$

Let $\varepsilon>0$. It follows from Theorem 3.2(b) that there exists $\beta_{0}=\beta_{0}(\varepsilon)$ such that $\left|\left(K_{\beta} \varphi_{\beta p_{j}}, \varphi_{\beta p_{j}}\right)-\hat{\kappa}\left(p_{j}\right)\right|<\varepsilon$ for $\beta \geq \beta_{0}, j \in Z$. Then, for $\beta \geq \beta_{0}$,

$$
\begin{gathered}
\frac{2 \pi}{\beta} \sum_{\lambda \in \sigma^{+}\left(K_{\beta}\right)} \lambda \geq \sum_{\substack{j \in Z \\
\hat{\kappa}\left(p_{j}\right) \geq 0 \\
\left|p_{j}\right| \leq P}}\left[\hat{\kappa}\left(p_{j}\right)-\varepsilon\right], \\
\frac{2 \pi}{\beta} \sum_{\lambda \in \sigma^{+}\left(K_{\beta}\right)} \lambda \geq \frac{2 \pi}{\beta} \sum_{\substack{j \in Z \\
\left|p_{j}\right| \leq P}} \hat{\kappa}_{+}\left(p_{j}\right)-\left(2 P_{+} \frac{2 \pi}{\beta_{0}}\right) \varepsilon .
\end{gathered}
$$

The first term on the right is a Riemann sum for the continuous function $\hat{\kappa}_{+}$on $[-P, P]$ with $\Delta p=2 \pi / \beta$. Thus, there exists $\beta_{1} \geq \beta_{0}$ such that

$$
\frac{2 \pi}{\beta} \sum_{\lambda \in \sigma^{+}\left(K_{\beta}\right)} \lambda \geq \int_{-P}^{P} \hat{\kappa}_{+}(p) d p-\left(4 P+\frac{4 \pi}{\beta_{0}}\right) \varepsilon
$$

for $\beta \geq \beta_{1}$. Since this inequality holds for any $\varepsilon>0$, it follows that

$$
\liminf _{\beta \rightarrow \infty} \frac{2 \pi}{\beta} \sum_{\lambda \in \sigma^{+}\left(K_{\beta}\right)} \lambda \geq \int_{-P}^{P} \hat{\kappa}_{+}(p) d p .
$$


Let $P \rightarrow \infty$ to obtain (a) in the theorem. Then (b) follows trivially. 口

Example 4.2. Let

$$
\kappa(u)= \begin{cases}1, & |u| \leq 1 \\ 0, & |u|>1\end{cases}
$$

Then

$$
\hat{\kappa}(p)= \begin{cases}2, & p=0 \\ 2 \frac{\sin p}{p}, & p \neq 0 .\end{cases}
$$

In this case, $\hat{\kappa} \notin L^{1}(R)$,

$$
\int_{-P}^{P} \hat{\kappa}_{+}(p) d p \rightarrow \infty \quad \text { as } \quad P \rightarrow \infty
$$

and Theorem 4.5 gives

$$
\sum_{\lambda \in \sigma^{+}\left(K_{\beta}\right)} \lambda \rightarrow \infty \quad \text { as } \beta \rightarrow \infty
$$

Example 4.3. Let $\kappa(u)=e^{-|u|}$, as in Example 4.1. In this case, Theorem 4.5 gives

$$
\liminf _{\beta \rightarrow \infty} \frac{2 \pi}{\beta} \sum_{\lambda \in \sigma^{+}\left(K_{\beta}\right)} \lambda \geq 2 \pi .
$$

5. Toeplitz forms. Further information about the distribution of the eigenvalues of $K_{\beta}$ is furnished by results on Toeplitz forms given in the book by Grenander and Szegö [4]; see also Kac, Murdock, and Szegö [6]. They work in a more restrictive setting:

$$
\kappa \in L^{2}(R), \quad \kappa(-u)=\overline{\kappa(u)}, \quad \hat{\kappa} \in L^{1}(R), \hat{\kappa} \text { bounded. }
$$

For $[a, b] \subset \sigma(K)$ with $0 \notin[a, b]$, let $N_{\beta}(a, b)$ be the number of eigenvalues $\lambda \in \sigma\left(K_{\beta}\right)$ with $a<\lambda<b$, where the eigenvalues are repeated as to multiplicity. Let $\nu$ denote Lebesgue measure on $R$. 
Theorem 5.1 [4, Theorem 8.6]. Assume $\kappa \in L^{2}(R), \kappa(-u)=\overline{\kappa(u)}$, $\hat{\kappa} \in L^{1}(R)$, and $\hat{\kappa}$ bounded. Let $a, b \in R$ such that $0 \notin[a, b]$ and

$$
\nu\{p: \hat{\kappa}(p)=a\}=0, \quad \nu\{p: \hat{\kappa}(p)=b\}=0 .
$$

Then

$$
\lim _{b \rightarrow \infty} \frac{N_{\beta}(a, b)}{\beta}=\frac{1}{2 \pi} \nu\{p: a<\hat{\kappa}(p)<b\} .
$$

Now suppose that $\kappa \in L^{1}(R), \kappa(-u)=\overline{\kappa(u)}$, and $\hat{\kappa} \in L^{1}(R)$. Then both $\kappa$ and $\hat{\kappa}$ are bounded and continuous. It follows that $\kappa \in L^{2}(R)$ and $\hat{\kappa} \in L^{2}(R)$. For example, the Picard kernel satisfies all these conditions, but Example 4.2 does not.

Theorem 5.2. Assume $\kappa \in L^{1}(R), \kappa(-u)=\overline{\kappa(u)}$ and $\hat{\kappa} \in L^{1}(R)$. Then $\sigma\left(K_{\beta}\right)$ is asymptotically dense in $\sigma(K)$ as $\beta \rightarrow \infty$.

Proof. A number $\lambda \in \sigma(K)$ will be called a regular point if $\nu\{p$ : $\hat{\kappa}(p)=\lambda\}=0$ and otherwise an irregular point. It is an easy exercise to show that the set of irregular points is countable. For example, if we define

$$
f(\lambda)=\nu\left\{\theta \in(-1,1): \hat{\kappa}\left(\tan \frac{\pi}{2} \theta\right)=\lambda\right\}, \quad \lambda \in \sigma(K),
$$

then $\lambda$ is irregular if and only if $f(\lambda)>0$. Since $f(\lambda) \leq 2$, there are at most two irregular points with $f(\lambda) \geq 1$, at most four with $f(\lambda) \geq 1 / 2$, and so on, which gives a countable ordering of the irregular points of $\sigma(K)$.

For any $\varepsilon>0$, the spectrum $\sigma(K)=[m, M]$ can be covered by a finite number of open intervals $I_{j}, j=1, \ldots, n$, each of length $\varepsilon$. If one or more endpoints happen to irregular, move each such endpoint so as to (a) make each endpoint a regular point; (b) reduce the length of the interval; (c) retain a covering of $\sigma(K)=[m, M]$.

Since the set of irregular points is countable, this is possible. By Theorem 5.1, there exists $\beta(\varepsilon)$ such that each interval $I_{j}$ contains at least one eigenvalue of $K_{\beta}$ for $\beta \geq \beta(\varepsilon)$. 
Example 5.1. For the Picard kernel $\kappa(u)=e^{-|u|}$, Theorem 5.1 gives

$$
\lim _{\beta \rightarrow \infty} \frac{N_{\beta}(a, b)}{\beta}=\frac{1}{\pi}\left[\left(\frac{2}{a}-1\right)^{1 / 2}\left(\frac{2}{b}-1\right)^{1 / 2}\right] \quad \text { for } 0<a<b<2 .
$$

This is consistent with Theorem 5.2.

\section{APPENDIX}

Let $A$ be a compact self-adjoint operator on a Hilbert space $H$. We shall estimate the number of positive eigenvalues of $A$ in terms of values of quadratic forms $\left(A x_{j}, x_{j}\right)$ with $\left\{x_{j}\right\}$ orthonormal. Lower bounds for leading eigenvalues are also given.

Assume that $A$ has at least one positive eigenvalue. Let $\sigma^{+}(A)=$ $\{\lambda \in \sigma(A): \lambda>0\}$. Then

$$
\sigma^{+}(A)=\left\{\lambda_{k}: k=1,2, \ldots\right\},
$$

where the positive eigenvalues are repeated as to multiplicity, and

$$
\lambda_{1} \geq \lambda_{2} \geq \cdots \geq \lambda_{k} \geq \cdots .
$$

Let $\# \sigma^{+}(A)=n$ if $\sigma^{+}(A)=\left\{\lambda_{1}, \ldots, \lambda_{n}\right\}$ and $\# \sigma^{+}(A)=\infty$ otherwise. If $\# \sigma^{+}(A)=\infty$, then $\lambda_{k} \rightarrow 0$ as $k \rightarrow \infty$.

There exist orthonormal eigenfunctions $\varphi_{k} \in H$ such that

$$
A \varphi_{k}=\lambda_{k} \varphi_{k}, \quad\left(A \varphi_{k}, \varphi_{k}\right)=\lambda_{k} .
$$

The eigenvalues $\lambda_{k}$ are the maximum values of Rayleigh quotients $(A x, x) /\|x\|^{2}$ on successive subspaces of $H$. In other words, the positive eigenvalues of $A$ are also the positive eigenvalues of the quadratic form $(A x, x)$ (see, for example, [12, Chapter 3]).

We shall make use of the following monotonicity property associated with the Rayleigh-Ritz method (see, for example, [12, Chapter 3, Theorem 7.1]). In this theorem and throughout the appendix, $A$ is compact, self-adjoint, and has at least one positive eigenvalue. The positive eigenvalues of $A$ are labelled as above. 
Theorem A1. Let $V$ be a subspace of $H$. Assume that on $V$ the quadratic form $(A x, x)$ has at least $n$ positive eigenvalues

$$
\mu_{1} \geq \mu_{2} \geq \cdots \geq \mu_{n}
$$

repeated as to multiplicity. Then $\# \sigma^{+}(A) \geq n$ and

$$
\lambda_{j} \geq \mu_{j} \quad \text { for } j=1, \ldots, n .
$$

If the subspace $V$ in the theorem is finite dimensional, then the eigenvalues $\mu_{j}$ are just the eigenvalues of the matrix $\left\{\left(A x_{j}, x_{k}\right)\right\}$, where $\left\{x_{j}\right\}$ is an orthonormal basis for $V$.

Lemma A1. Let $\left\{x_{j}: j=1, \ldots, n\right\}$ be an orthonormal set in $H$. Let the eigenvalues of the matrix $\left\{\left(A x_{j}, x_{k}\right): j, k=1, \ldots, n\right\}$ be $\mu_{1} \geq \mu_{2} \geq \cdots \geq \mu_{n}$, repeated as to multiplicity. Assume that exactly $n_{0}$ of these eigenvalues are positive. Then

(a) $\# \sigma^{+}(A) \geq n_{0}$,

(b) $\lambda_{j} \geq \mu_{j}$ for $j=1, \ldots, n_{0}$,

(c) $\sum_{j=1}^{n_{0}} \lambda_{j} \geq \sum_{j=1}^{n}\left(A x_{j}, x_{j}\right)$.

Proof. Both (a) and (b) follow directly from Theorem A1. Part (c) follows from

$$
\sum_{j=1}^{n_{0}} \lambda_{j} \geq \sum_{j=1}^{n_{0}} \mu_{j} \geq \sum_{j=1}^{n} \mu_{j}=\sum_{j=1}^{n}\left(A x_{j}, x_{j}\right) .
$$

An immediate consequence is

Corollary A1. Let $\left\{x_{j}: j=1, \ldots, n\right\}$ be an orthonormal set in $H$. Then

(a) $\sum_{\lambda \in \sigma^{+}(A)} \lambda \geq \sum_{j=1}^{n}\left(A x_{j}, x_{j}\right)$,

(b) $\sum_{j=1}^{n} \lambda_{j} \geq \sum_{j=1}^{n}\left(A x_{j}, x_{j}\right)$, if $\# \sigma^{+}(A) \geq n$. 
Theorem A2. Assume that there exists an orthonormal set $\left\{x_{j}: j=1, \ldots, n\right\}$ in $H$, with $n \geq 1$, such that

$$
\left(A x_{j}, x_{j}\right)>\frac{n-1}{n} \lambda_{1} \quad \text { for } j=1, \ldots, n,
$$

or the weaker condition

$$
\sum_{j=1}^{n}\left(A x_{j}, x_{j}\right)>(n-1) \lambda_{1} .
$$

Then

(a) $\# \sigma^{+}(A) \geq n$,

(b) $\lambda_{j}>[(n-j) /(n-j+1)] \lambda_{1}$ for $j=1, \ldots, n$.

Proof. From the weaker hypothesis and Corollary A1(a),

$$
\sum_{\lambda \in \sigma^{+}(A)} \lambda>(n-1) \lambda_{1},
$$

which cannot hold if $\# \sigma^{+}(A)<n$. Hence, $\# \sigma^{+}(A) \geq n$ and (a) is established. Consider (b). For $j=2, \ldots, n$, we have

$$
(j-1) \lambda_{1} \geq \sum_{k=1}^{j-1} \lambda_{k}, \quad(n-j+1) \lambda_{j} \geq \sum_{k=j}^{n} \lambda_{k} .
$$

By Corollary A1(b) and the weaker hypothesis of the theorem,

$$
\begin{aligned}
(j-1) \lambda_{1}+(n-j+1) \lambda_{j} & \geq \sum_{k=1}^{n} \lambda_{k} \geq \sum_{k=1}^{n}\left(A x_{k}, x_{k}\right) \\
& >(n-1) \lambda_{1}
\end{aligned}
$$

which implies (b).

Theorem A3. Let $\left\{x_{j}: j=1,2, \ldots\right\}$ be an orthonormal set in $H$. Then

$$
\sum_{\lambda \in \sigma^{+}(A)} \lambda>\sum_{j=1}^{\infty}\left(A x_{j}, x_{j}\right) .
$$


Proof. This follows by letting $n \rightarrow \infty$ in Corollary A1(a).

Acknowledgments. We are indebted to R. Kress for communicating an argument of P. Hähner and R. Kress for the equality of the spectra of $K_{\beta}$ on $C[0, \beta]$ and $L^{2}(0, \beta)$, and to H.F. Weinberger for valuable comments on the symmetric eigenvalue problem.

This work was supported in part by the U.S. Army Research Office through the Mathematical Sciences Institute at Cornell University, and in part by the Australian Research Council.

\section{REFERENCES}

1. P.M. Anselone, Collectively compact operator approximation theory and applications to integral equations, Prentice-Hall, Englewood Cliffs, N.J., 1971.

2. P.M. Anselone and I.H. Sloan, Integral equations on the half-line, J. Integral Equations Appl. 9 [supp.] (1985), 3-23.

3. _ Spectral approximations for Wiener-Hopf operators, J. Integral Equations Appl. 2 (1990), 237-261.

4. U. Grenander and G. Szegö, Toeplitz forms and their applications, 2nd ed., Chelsea Publ. Co., New York, 1984.

5. K. Jörgens, Linear integral operators, Pitman, Boston, 1982.

6. M. Kac, W.L. Murdock and G. Szegö, On the eigen-values of certain Hermitian forms, Arch. Rational Mech. Anal. 2 (1953), 707-800.

7. M.G. Krein, Integral equations on a half-line with kernel depending on the difference of the arguments, Amer. Math. Soc. Transl. 22 (1963), 163-288.

8. M.G. Krein and M.A. Rutman, Linear operators leaving invariant a cone in a Banach space, Amer. Math. Soc. Transl. 26 (1948), 3-95.

9. R. Kress, Linear integral equations, Springer-Verlag, Berlin, 1989.

10. E.M. Stein and G. Weiss, Introduction to Fourier analysis on Euclidean spaces, Princeton Univ. Press, 1971.

11. B. Silbermann, Numerical analysis for Wiener-Hopf integral equations in spaces of measurable functions, Seminar analysis, Akad. Wiss. DDR, Berlin, 1986.

12. H.F. Weinberger, Variational methods for eigenvalue approximations, SIAM, Philadelphia, 1974.

13. W.L. Wendland, Die Fredholmsche Alternative für Operatoren, die bezüglich eines bilinearen Funktionals adjungiert sind, Math. Z. 101 (1967), 61-64.

Department of Mathematics, Oregon State University, Corvallis, OR 97331

School of Mathematics, University of New South Wales, Sydney, NSW 2033 Australia 